\title{
Fourier Transform Ion Cyclotron Resonance Detection of Multiphoton Ionization Spectroscopy
}

\author{
Seung Koo Shin, Seung-Jin Han, and Byungjoo Kim \\ Department of Chemistry, University of California, Santa Barbara, Califorria, USA
}

\begin{abstract}
Fourier transform ion cyclotron resonance (FT-ICR) detection was tested for resónanceenhanced multiphoton ionization (REMPI) spectroscopy. The $(2+1)$ REMPI spectra of acetaldehyde were obtained in the wavelength range $364-354 \mathrm{~nm}$ via a two-photon resonant $3 s \leftarrow n$ Rydberg transition. The space-charge effects on the REMPI spectra were examined in the vicinity of the $0_{0}^{0}$ transition. The trapping efficiency measurement shows that all the ions produced from REMPI dissociation processes are arrested in the ion cyclotron resonance cell even in the presence of space-charge interactions. Axial kinetic energy release distributions of ions were extracted from the trapping efficiency data obtained under a new space-charge-free condition. FT-ICR peak heights were measured as a function of pressure at different laser powers, magnetic field strengths, and ion excitation methods to test for the detection linearity. The FT-ICR detection responds linearly to the number of ions in a low pressure limit. The product branching ratio was measured by using various ion excitation methods and was compared with the previous quadrupole mass spectrometric study. FT-ICR detection yields the mass-selected REMPI spectra and the product branching ratio in the absence of kinetic shifts. (J Am Soc Mass Spectrom 1996, 7, 1018-1025)
\end{abstract}

$\mathrm{M}$ ultiphoton ionization (MPI) is one of the most widely used physical methods for spectroscopic studies of resonant intermediate states [1-6]. For mass spectroscopic studies, a time-of-flight mass spectrometer (TOF-MS) typically has been employed owing to its ease to obtain a complete mass spectrum from each ionizing pulse [7-11]. It allows the accurate determination of molecular weights [12] and kinetic energy release distributions of ions from metastable ion decompositions [13]. However, ion collection efficiencies vary with the extraction ion optics, and product branching ratios are subject to the kinetic shifts of ion signals [14]. Sector and quadrupole mass spectrometers also have been used for mass-selective studies of multiphoton ionization processes [15], but their applications have been limited by a poor duty cycle, which requires several laser shots per atomic mass unit. A Fourier transform ion cyclotron resonance (FT-ICR) spectrometer has been used to obtain MPI mass spectra from each ionizing pulse [16-19]. The ion trapping helps to accumulate and detect all ions produced from MPI processes.

Most optical spectroscopic studies have been carried out by using a parallel plate detector that probes a total ion current as a function of excitation wavelength

Address reprint requests to Seung Koo Shin, Department of Chemistry, University of California, Santa Barbara, CA 93106-9510.
[20]. The TOF-MS or the quadrupole mass spectrometers often are employed to obtain the mass-selected MPI spectra as a function of wavelength [15, 21, 22]. However, presently there are no reports of mass-selected optical MPI spectroscopy that uses FT-ICR spectrometry. All previous studies focused on ionization of the selected target molecules in the ion cyclotron resonance (ICR) cell [16-19, 23-26]. For instance, McIver and co-workers [16] obtained two-photon ionization (2PI) mass spectra of polycyclic aromatic hydrocarbons (PAHs) from a single laser shot at 248 and $222 \mathrm{~nm}$. Carlin and Freiser [17] reported the selective 2PI of PAHs at $266 \mathrm{~nm}$. Gross and co-workers [18] used 2PI to detect PAHs in a gas chromatography-Fourier transform-ion cyclotron resonance detection system. Eyler and co-workers [19] performed MPI of laserdesorbed nonvolatile samples. Most recently, Bowers and co-workers [23-26] employed the MPI for stateselective generation of $\mathrm{NO}^{+}\left(\mathrm{X}^{1} \Sigma^{+}, v=1-5\right)$.

In the present study, the resonance-enhanced MPI (REMPI) spectra of acetaldehyde were taken in the ICR cell to examine the performance of FT-ICR detection for REMPI spectroscopy. MPI in the ICR cell that uses a focused pulsed laser beam is considered to be a pulsed near-point ion source located in the middle of the ICR cell. MPI mass spectra were collected as a function of wavelength in the range 364-354 $\mathrm{nm}$ and the mass-selected REMPI spectra of acetaldehyde were obtained therefrom. 
The REMPI processes employed in this work involve a two-photon resonant $3 s \leftarrow n$ Rydberg transition of acetaldehyde followed by one-photon ionization [15, 20, 21, 27-31]. The (2+1) REMPI spectra of acetaldehyde first was reported in the wavelength range 368-358 $\mathrm{nm}$ by Heath et al. [27], who used a parallel-plate ionization cell. Fisanick et al. [15] reported both MPI mass spectra and mass-selected REMPI spectra by using a quadrupole mass filter. Goodman and co-workers [30] studied torsional vibronic bands by using a parallel plate detector. Recently, the mass-selected REMPI spectra were recorded by Buntine et al. [21] for several deuterated acetaldehydes for the differentiation of possible sym and asym rotamers of the methyl group by utilizing a TOF detection system. Most recently, Pfab and co-workers [20] reported the REMPI spectrum of jet-cooled acetaldehyde over the extended wavelength range 365-352 nm by using a parallel-plate detector.

The performance tests focus on the trapping efficiency, the detection linearity, and the product branching ratio. The trapping efficiency was measured by comparing mass spectral peak heights as a function of trapping voltage. This measurement provides axial kinetic energy release distributions (AKERDs) of ions. The detection linearity was examined by monitoring mass spectral peak heights as a function of sample pressure. The product branching ratio was used to test the overall performance of FT-ICR detection, because it depends on both the trapping efficiency and the detection linearity. A single resonant radiofrequency ( $\mathrm{rf}$ ) excitation method was compared with broadband excitation methods to check the mass discrimination due to ion excitation. The product branching ratio obtained from the pulsed experiment was compared with that from the bulk experiment to examine the mass discrimination due to collisional damping of transient signals.

\section{Experimental}

Experiments were performed on the pulsed beam ICR setup shown in Figure 1a, which was interfaced with an IonSpec (Irvine, CA) Omega-386 Fourier transform (FT) data system. A general description of the experimental setup has been given elsewhere [23]. Experimental procedures for the REMPI spectroscopy in the ICR cell will be outlined briefly in subsequent text.

An ICR chamber is operated under a base pressure of $5 \times 10^{-8}$ torr. A molecular beam of neat acetaldehyde is pulsed into a differentially pumped chamber for $\sim 100 \mu \mathrm{s}$. The molecular beam enters the ICR chamber through a 1.3-mm-diameter orifice skimmer. Output from an excimer pumped dye laser is focused onto the center of a 1.85 -in. cubic ICR cell by a $50-\mathrm{mm}$ focal length lens. The laser beam intersects the molecular beam in the center of the ICR cell. A magnetic field axis is perpendicular to both the molecular beam and the laser propagation axes, whereas the laser polariza-

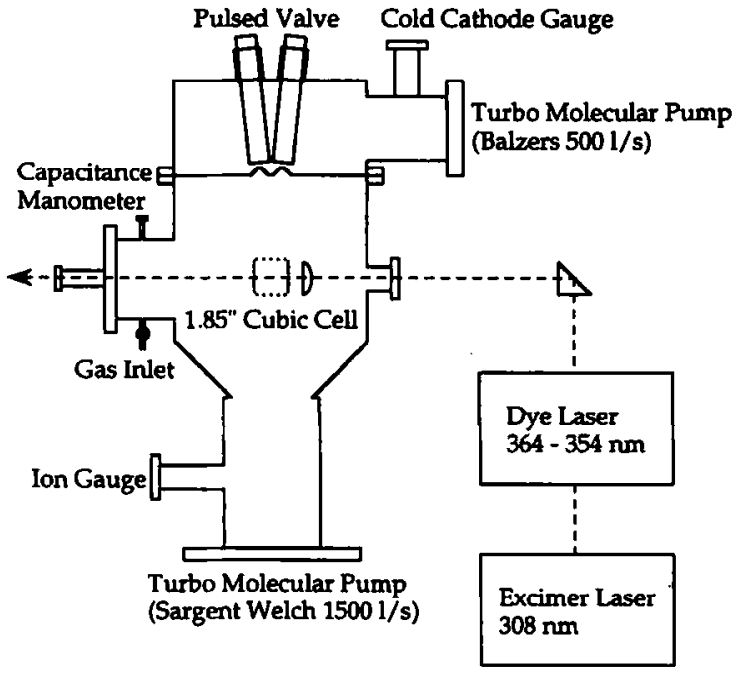

(a)

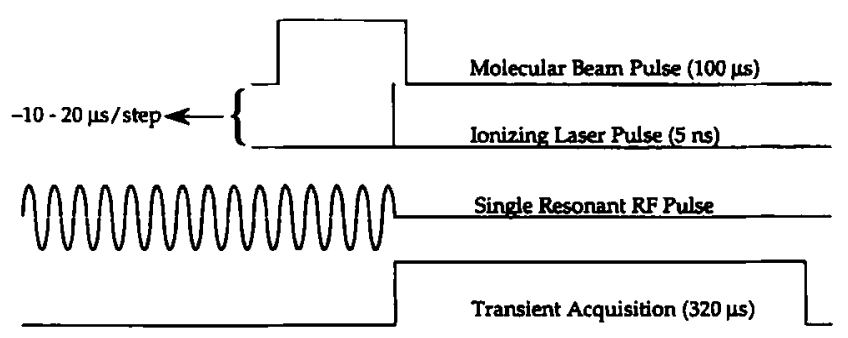

(b)

Figure 1. (a) A pulsed molecular beam ICR setup and (b) a pulse sequence for the single resonant $\mathrm{rf}$ excitation experiment. The molecular beam and laser pulses are scanned together in a 10-20- $\mu$ s decrement step.

tion is linear and perpendicular to the magnetic field axis. The trapping and detector plates are made out of 0.020-in.-thick molybdenum and the transmitter plates are composed of $90 \%$ transmission stainless steel mesh. Each of the detector plates has a 3/8-in.-diameter hole to pass the laser beam horizontally to the center of ICR cell, and the transmitter plates are located on the top and bottom of the cell to allow the passage of the molecular beam. A time delay between the pulsed valve and the pump laser was optimized to give the best signal-to-noise ratio while minimizing ionmolecule reactions. Since the proton-transfer reactions of $\mathrm{HCO}^{+}$with the parent neutral that form the protonated acetaldehyde ion are nearly collision-limited [31], collision-free conditions are achieved by minimization of the protonated ion signal in the mass spectrum. The pressure dependence experiments were performed by flowing the sample through a leak valve. The sample pressure was monitored with an ion gauge mounted near the ICR cell.

Ions were detected by an impulse or a broadband rf chirp excitation method. Image charge signals induced on the receiver plates were processed and digitized by a 2-MHz, 32K data point, 9-bit transient digitizer. A single resonant if excitation method was applied for 
the branching ratio measurement. Figure $1 \mathrm{~b}$ shows the time sequence of the pulsed valve, pump laser, rf excitation, and detection trigger controlled by a digital delay generator (Stanford Research DDG 535, Sunnyvale, CA). The duration of the single resonant rf burst was increased gradually by $1-20-\mu$ s increments from the minimum duration of at least one resonant cycle. The start trigger of the transient digitizer was synchronized to the falling edge of the $\mathrm{rf}$ pulse. A delay between the end of $\mathrm{rf}$ burst and the detection trigger st art was less than $1 \mu \mathrm{s}$. A transient ICR signal was digitized for $320 \mu$ s with a 100 megasamples per second transient digitizer on a CAMAC crate.

A DMQ dye was used to scan the 364-354-nm wavelength range with a $0.15-\mathrm{cm}^{-1}$ increment. A bandwidth of the dye laser is $\sim 0.35 \mathrm{~cm}^{-1}$ and the pulse width is $\sim 5 \mathrm{~ns}$ with a maximum pulse energy of $\sim 30 \mathrm{~mJ}$. The FT mass spectrum at each wavelength is an average of four laser shots.

\section{Results and Discussion}

\section{Multiphoton Ionization Spectrum of Acetaldehyde}

Figure 2 shows an MPI mass spectrum of jet-cooled acetaldehyde from a single laser shot at the Rydberg transition origin. The MPI of acetaldehyde yields the parent ion as well as its fragments $\mathrm{HCO}^{+}$and $\mathrm{CH}_{3} \mathrm{CO}^{+}$. Mass resolutions are Fourier transform limited: 5600 for $\mathrm{HCO}^{+}$and 3700 for $\mathrm{CH}_{3} \mathrm{CO}^{+}$. A threephoton energy $(\lambda=363.48 \mathrm{~nm}) 10.23 \mathrm{eV}$ is very close to the ionization potential $10.229 \mathrm{eV}$ [32-34]. The daughter ions are derived from one-photon excitation of the parent ion to the $\tilde{B}^{2} \mathrm{~A}^{\prime}$ state [31]. The $\alpha \mathrm{C}-\mathrm{C}$ cleavage to form $\mathrm{HCO}^{+}$with a loss of $\mathrm{CH}_{3}$ is a dominant product channel. Although the formation of $\mathrm{CH}_{3}^{+}$from the $\tilde{\mathrm{B}}^{2} \mathrm{~A}^{\prime}$ state is energetically accessible with a loss of $\mathrm{HCO}\left(\tilde{\mathrm{X}}^{2} \mathrm{~A}^{\prime}\right)$, no $\mathrm{CH}_{3}^{+}$ions are observed. The $\alpha \mathrm{C}-\mathrm{H}$ cleavage to yield $\mathrm{CH}_{3} \mathrm{CO}^{+}$with a loss of $\mathrm{H}$ is a minor channel. The absence of fragment ions such as $\mathrm{CH}_{4}^{+}, \mathrm{CH}_{3}^{+}$, and $\mathrm{CH}_{2}^{+}$is particularly intriguing

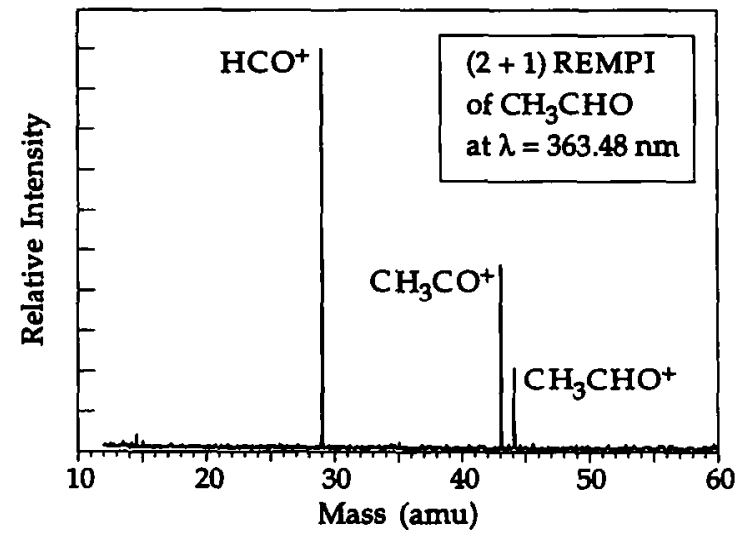

Figure 2. A mass spectrum of acetaldehyde obtained at the origin of the two-photon resonant $3 s \leftarrow n$ Rydberg transition $(\lambda=363.48 \mathrm{~nm})$. The trapping voltage was $1.50 \mathrm{~V}$ and the mag netic field strength was $0.76 \mathrm{~T}$. because they have been found in the electron impact spectra as well as in the previous MPI mass spectra obtained by using a quadrupole mass filter [15]. Fisanick et al. proposed a sequential stepwise mechanism for such fragments. It is speculated that the difference in fragmentation between the present result and the previous experiment is due to the presence of a magnetic field that affects the dissociation dynamics in the excited state.

The mass-selected REMPI spectra of acetaldehyde are shown in Figure 3a for $\mathrm{HCO}^{+}$and Figure 3b for $\mathrm{CH}_{3} \mathrm{CO}^{+}$. They were obtained by ionizing a pulsed molecular beam of neat acetaldehyde with a $\sim 15$ $\mathrm{mJ} /$ pulse laser power. The two daughter ions exhibit almost identical spectral features over the entire wavelength range. The vibrational assignments given in Figure $3 \mathrm{a}$ are based on the vacuum-ultraviolet spectral assignments by Creighton and Bell [35]. The strong bands such as $0_{0}^{0}$ and $10_{0}^{1}$ display anomalous band shapes due to the space-charge effects. The rotational temperature of acetaldehyde cannot be estimated because of the power broadening. The product branching
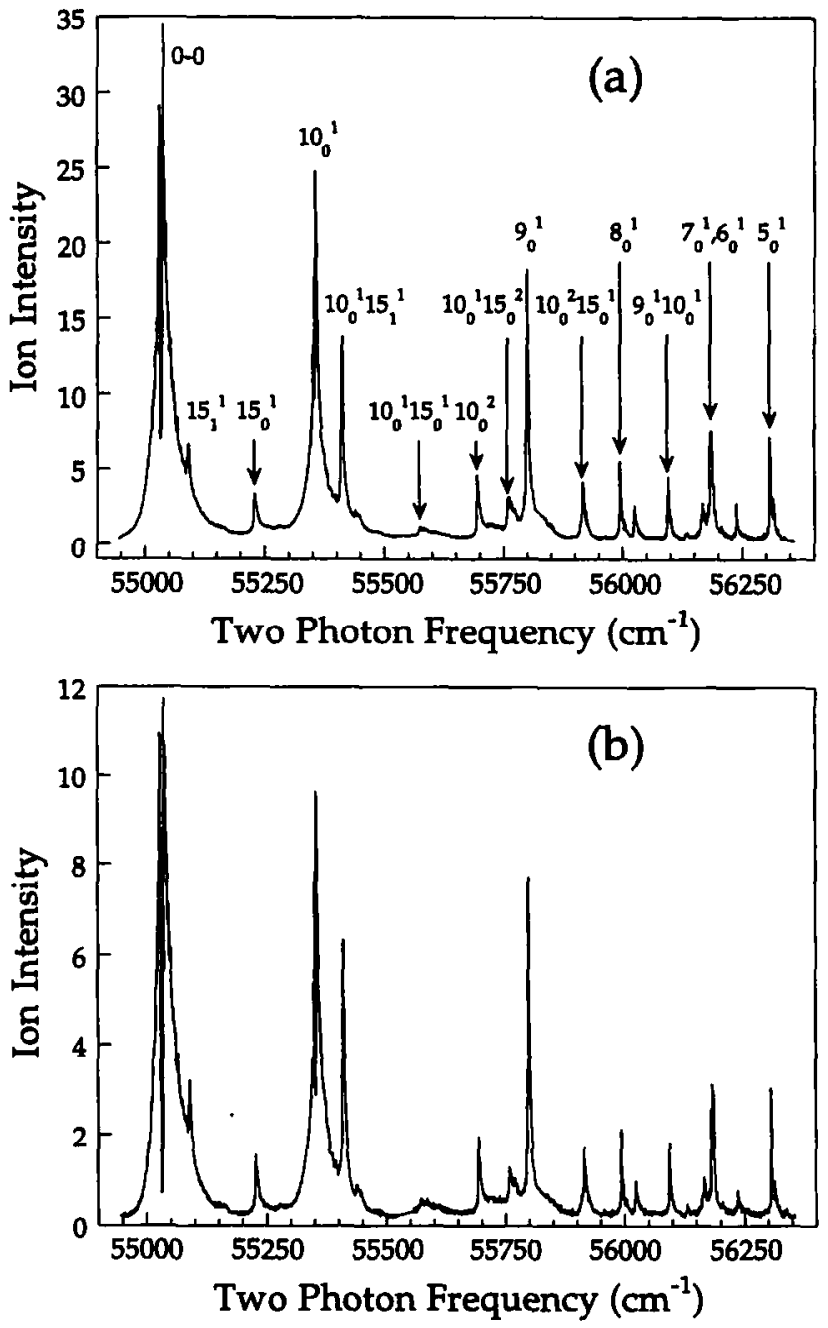

Figure 3. The mass-selected $2+1$ REMPI spectra of acetaldehyde with vibrational assignments [35]: (a) $\mathrm{HCO}^{+}$and (b) $\mathrm{CH}_{3} \mathrm{CO}^{+}$. The laser power was $15 \mathrm{~mJ} /$ pulse. 
ratio $\left[\mathrm{HCO}^{+}\right] /\left[\mathrm{CH}_{3} \mathrm{CO}^{+}\right]=\sim 2.1 \pm 0.2$ remains nearly constant over the entire wavelength range and mostly independent of vibrational excitations.

\section{Space-Charge Effects}

When a dense molecular beam is ionized by a high power laser, FT-ICR signals are affected strongly by instantaneous Coulomb interactions among ions. The Coulomb explosion induces large amplitude oscillations of ions in the ICR cell that deteriorate the detection linearity (Han, S-J.; Shin, S. K., unpublished). The space-charge effect in the pulsed molecular beam experiment was studied further by examination of the laser power dependence of the band shape in the vicinity of the $0_{0}^{0}$ transition. The ion density is proportional to the product of multiphoton absorption cross sections. Provided that the one-photon ionization cross section remains constant, the ion density is determined by a two-photon absorption cross section. Thus, the band shape represents rotational contours of twophoton transition by a linearly polarized light, which consists mainly of a strong $Q$ branch $(\Delta J=0)$ with $\Delta K=0, \pm 2$ in the near-prolate top limit. Figure $4 \mathrm{a}$ and $\mathrm{b}$ shows the REMPI spectra of the $0_{0}^{0}$ band obtained at 15 and $6 \mathrm{~mJ} /$ pulse, respectively. The anomalous band shape shown in Figure 4a illustrates the space-charge effects that decrease the peak height and deteriorate the mass resolution near the band center. No such effects appear in Figure $4 \mathrm{~b}$ at the low laser power, which indicates that the space-charge interactions are attenuated greatly. All performance tests were carried out under the low ion density condition.

\section{Trapping Efficiency}

Figure 5a shows FT-ICR peak heights as a function of trapping voltage with the excitation wavelength fixed at the red wing of the $0_{0}^{0}$ band, whereas Figure $5 \mathrm{~b}$ shows the trapping data obtained at the band center. The laser power was fixed at $6 \mathrm{~mJ} /$ pulse. In Figure $5 \mathrm{a}$, the parent ions were arrested readily by a near-zero trapping voltage, which indicates negligible Coulomb interactions among ions. The daughter ions were arrested at higher trapping voltages than the parent ion. The complete trapping of $\mathrm{HCO}^{+}$requires higher potentials than $\mathrm{CH}_{3} \mathrm{CO}^{+}$. The total ion intensity increases with increasing trapping voltage until all ions are trapped in the ICR cell.

When the wavelength was tuned to the band center as shown in Figure $5 b$, the trapping efficiency curves were shifted to higher voltages. Because the kinetic energy release from photodissociation is independent of laser power, the apparent shift in the trapping efficiency curve presumably is due to instantaneous Coulomb interactions among ions. It appears that the trapping efficiency data are more sensitive to space-charge interactions than the FT-ICR peak height and width. In spite of space-charge interactions, all
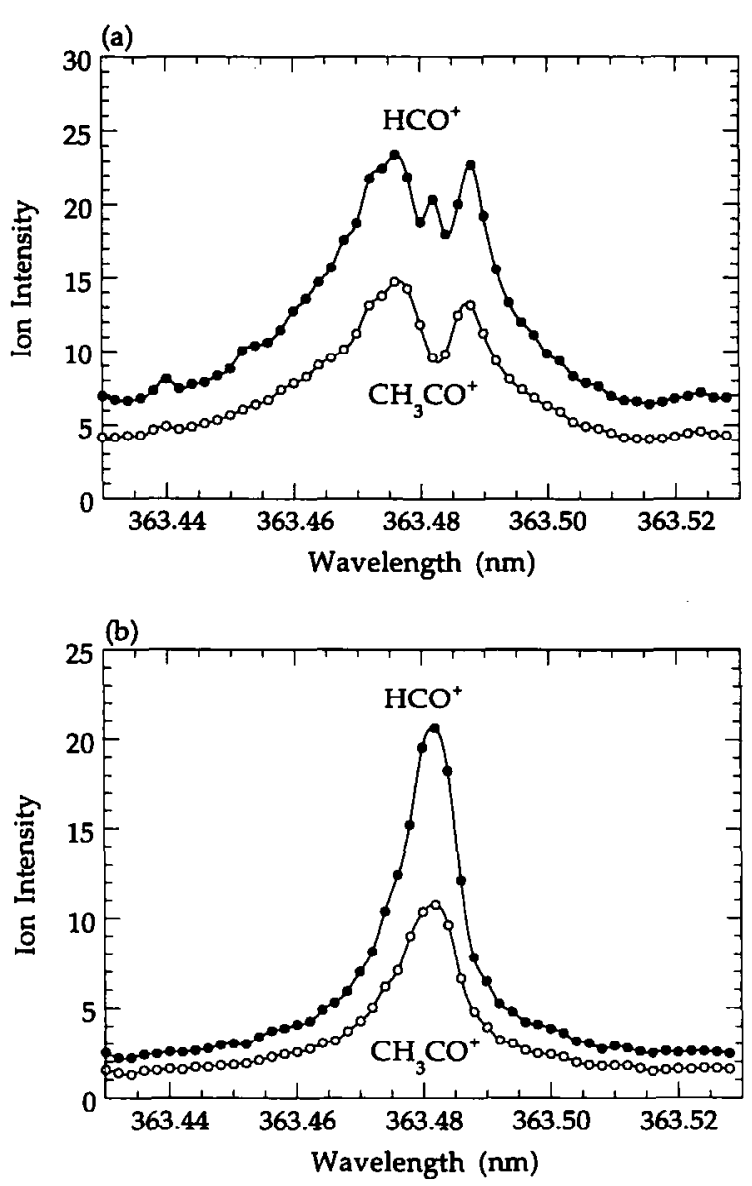

Figure 4. Intensities of $\mathrm{HCO}^{+}$and $\mathrm{HC}_{3} \mathrm{CO}^{+}$ions as a function of excitation wavelength. (a) $15 \mathrm{~mJ} /$ pulse and (b) $6 \mathrm{~mJ} / \mathrm{pulse}$. The magnetic field strength was $0.76 \mathrm{~T}$ and the trapping voltage was $1.50 \mathrm{~V}$. A pulsed molecular beam of neat acetaldehyde was used.

ions were arrested. Thus, a near-unity ion collection efficiency is accomplished in the ICR ion trap, which allows the unbiased measurement of product branching ratios in the absence of kinetic shifts. In the timeof-flight or quadrupole mass detection, the ion extraction within a few microseconds after ionization introduces a bias against the product ions formed from slow metastable decompositions [36]. This kinetic shift of ion intensity due to the ion extraction field is absent in the ICR detection. The near-unity ion collection efficiency in the absence of the ion extraction stage is one of the unique features of ICR detection that may be useful for studies of REMPI dissociation processes in the absence of kinetic shifts and for studies of ion-molecule reactions of state-selected ions [31].

\section{Axial Kinetic Energy Release Distributions}

Because a collimated molecular beam propagates to a direction perpendicular to the magnetic field axis, the $(2+1)$ REMPI produces the parent ion with almost no axial kinetic energy. On the other hand, photofragmentations can release excess energies into the axial kinetic 

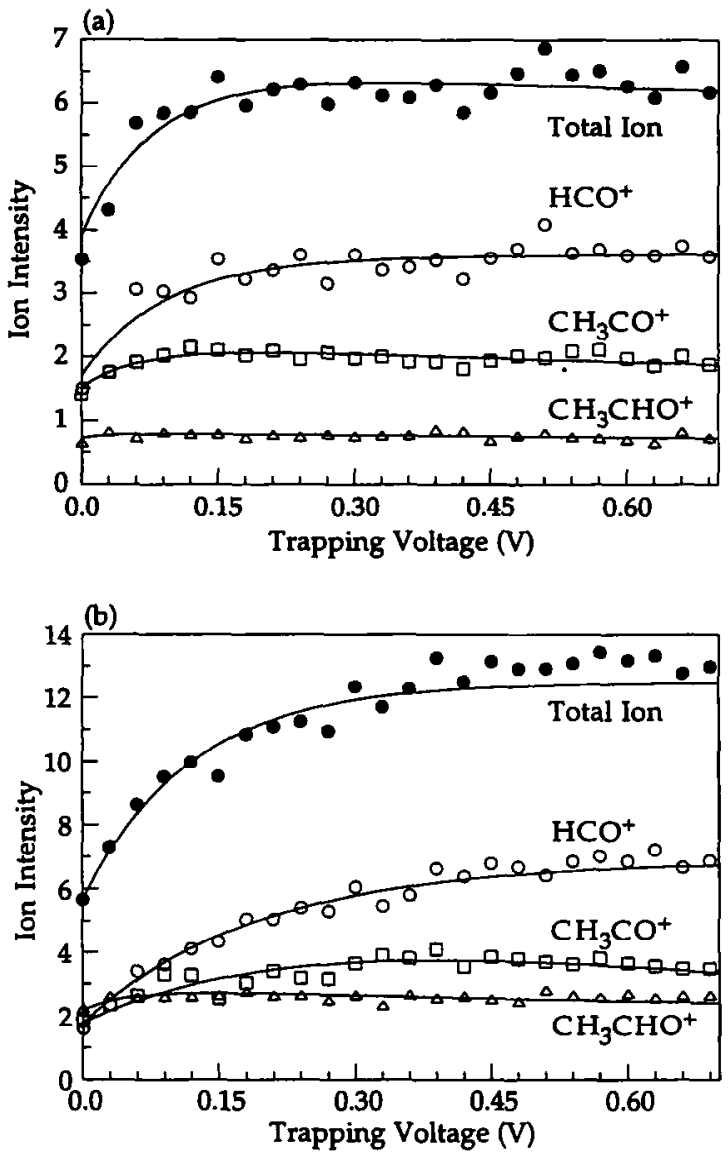

Figure 5. The trapping efficiency data as a function of trapping voltage. The laser wavelength was tuned to (a) the red wing of the $0_{0}^{0}$ band (low ion density) and (b) the band center (high ion density). The laser power was fixed at $7 \mathrm{~mJ} /$ pulse and the peak pressure in the ICR chamber from the pulsed molecular beam was $\sim 6 \times 10^{-6}$ torr.

energies of the daughter ions. AKERDs may be extracted from the trapping efficiency data [37, 38]. Figure 6 shows AKERDs of the daughter ions extracted from the space-charge-free data shown in Figure 5a. Average axial kinetic energies are estimated from

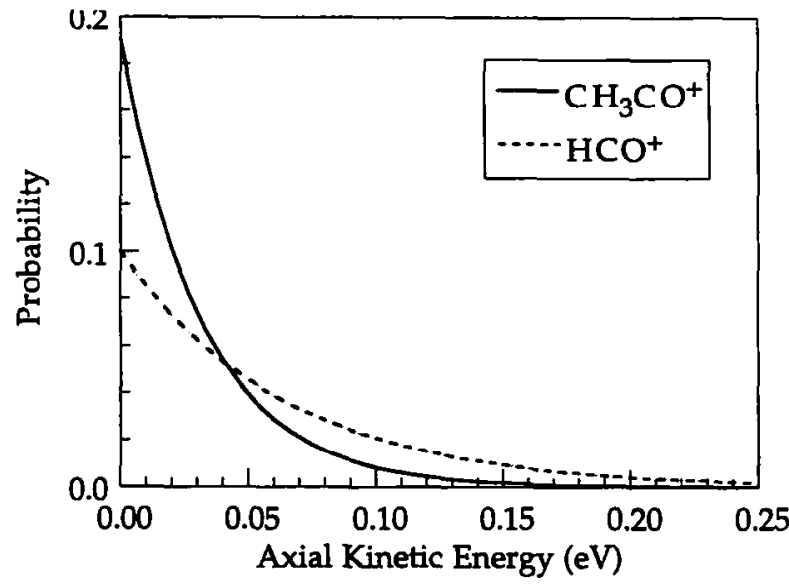

Figure 6. Axial kinetic energy distributions of ions extracted from the trapping efficiency data shown in Figure 4a.
AKERDs: $\sim 30 \mathrm{meV}$ for $\mathrm{CH}_{3} \mathrm{CO}^{+}$and $\sim 60 \mathrm{meV}$ for $\mathrm{HCO}^{+}$

It would be informative to compare average axial kinetic energy releases with kinematic expectation values. The total translational energy $E_{\mathrm{t}}$ available to the photofragments is given approximately by eq 1 :

$$
E_{\mathrm{t}}=E(h \nu)-\mathrm{D}(\mathrm{C}-\mathrm{X})-E_{\mathrm{int}}
$$

where $E(h \nu)$ is the photon energy, $E(363.48 \mathrm{~nm})=$ $3.41 \mathrm{eV}, \mathrm{D}(\mathrm{C}-\mathrm{X})$ is the $\mathrm{C}-\mathrm{X}$ bond dissociation enthalpy, $\mathrm{D}\left(\mathrm{CH}_{3} \mathrm{CO}^{+}-\mathrm{H}\right)=0.51 \mathrm{eV}$ and $\mathrm{D}\left(\mathrm{HCO}^{+}-\mathrm{CH}_{3}\right)=1.55 \mathrm{eV}$ [39], and $E_{\text {int }}$ is the internal energy of the fragments. The kinematic partitioning of total translational energy between photofragments yields the maximum translational kinetic energies of $66 \mathrm{meV}$ for $\mathrm{CH}_{3} \mathrm{CO}^{+}$and $634 \mathrm{meV}$ for $\mathrm{HCO}^{+}$. When compared with average axial kinetic energies, the maximum expectation value for the translational energy of $\mathrm{HCO}^{+}$is about 10 times greater than the average axial kinetic energy, whereas that of $\mathrm{CH}_{3} \mathrm{CO}^{+}$ is on the same order of magnitude. This comparison indicates that $\mathrm{HCO}^{+}$is translationally quite cold, but internally hot. This conclusion is corroborated by proton-transfer bracketing experiments that reveal the extent of internal excitation in $\mathrm{HCO}^{+}(\mathrm{Kim}, \mathrm{B}$.; Shin, S. $\mathrm{K}$., unpublished). The proton affinity (PA) of $\mathrm{CO}$ is $141.9 \mathrm{kcal} / \mathrm{mol}[40,41]$. (Values of proton affinities are corrected by anchoring the proton affinity of $\mathrm{CO}$ as $141.9 \mathrm{kcal} / \mathrm{mol}$.) $\mathrm{HCO}^{+}$from the MPI underwent endothermic proton-transfer reactions with $\mathrm{HBr}(\mathrm{PA}=$ $139.3 \mathrm{kcal} / \mathrm{mol})$ [40], $\mathrm{N}_{2} \mathrm{O}(\mathrm{PA}=137.8 \mathrm{kcal} / \mathrm{mol})$ [41], and $\mathrm{HCl}(\mathrm{PA}=133.5 \mathrm{kcal} / \mathrm{mol})[40]$, but no protontransfer products were observed with $\mathrm{CH}_{4}(\mathrm{PA}=130.2$ $\mathrm{kcal} / \mathrm{mol})$ [41] and $\mathrm{NO}(\mathrm{PA}=127.5 \mathrm{kcal} / \mathrm{mol})$ [40]. The internal energy of $\mathrm{HCO}^{+}$is estimated as high as $\sim 10 \pm 1.5 \mathrm{kcal} / \mathrm{mol}$.

The kinetic energy of the magnitude considered here is not measured easily by either TOF-MS or quadrupole mass spectrometry because of uncertainty in the ion energy deconvolution. FT-ICR detection affords both the low kinetic energy measurement owing to the absence of ion extraction field and the internal energy measurement thanks to the ion trapping capability.

\section{Detection Linearity}

To examine the dependence of the FT-ICR signal on the number of ions in the ICR cell, FT-ICR peak heights were measured. as a function of pressure at two different laser powers (7 and $20 \mathrm{~mJ} / \mathrm{pulse})$ and at two different magnetic field strengths (0.76 and $1.1 \mathrm{~T}$ ). The pressure variation allows one to control the number of ions in an ionizing volume. Figure $7 \mathrm{a}$ and $\mathrm{b}$ shows the results obtained at 0.76 and $1.1 \mathrm{~T}$, respectively. Both the impulse and broadband rf chirp excitation methods gave almost identical results at two different laser powers. At $0.76 \mathrm{~T}, \mathrm{FT}-\mathrm{ICR}$ peak heights increase lin- 

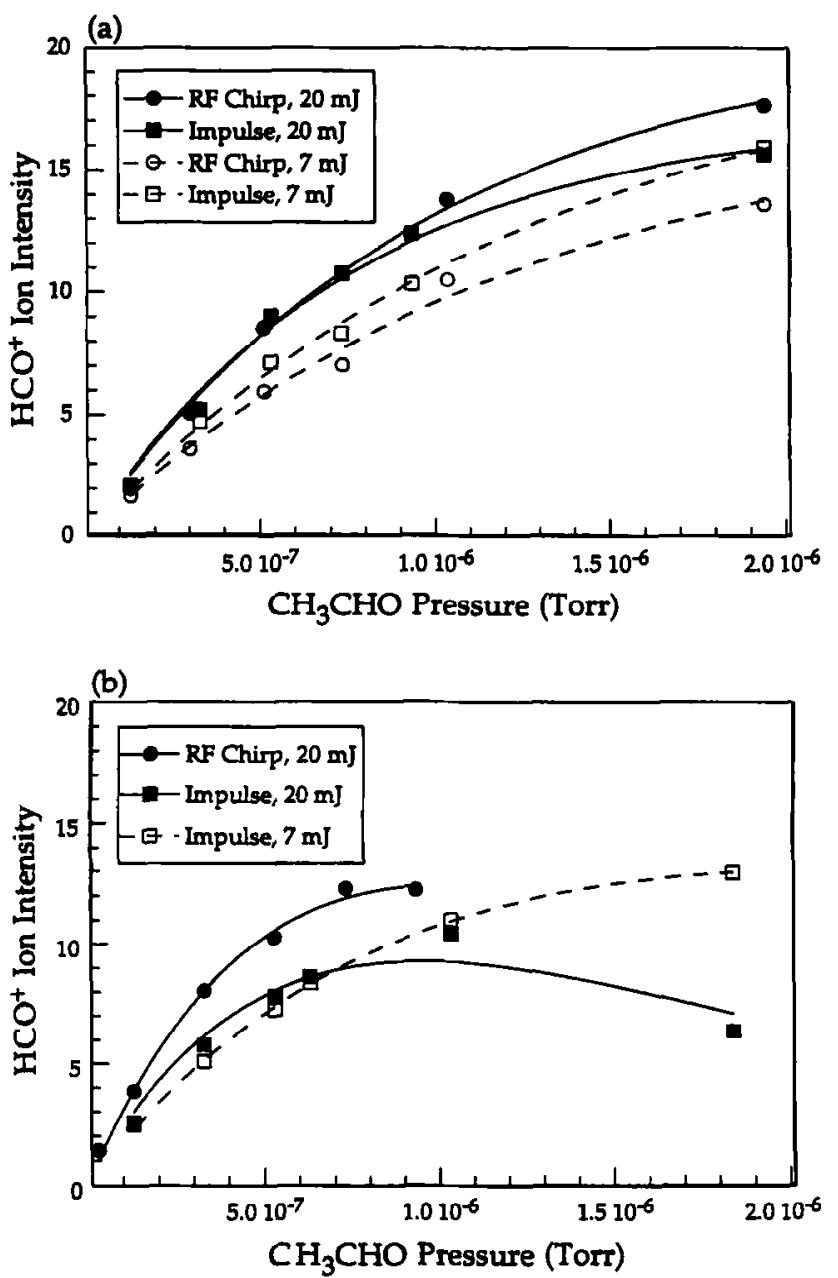

Figure 7. The pressure dependence of the $\mathrm{HCO}^{+}$ion intensity at two different laser powers, magnetic field strengths, and excitation methods. (a) $\mathrm{B}=0.76 \mathrm{~T}$ and (b) $\mathrm{B}=1.1 \mathrm{~T}$. The laser wavelength was $363.48 \mathrm{~nm}$ and the trapping voltage was $1.50 \mathrm{~V}$.

early in the low pressure range $1.0-5.0 \times 10^{-7}$ torr. However, they deviate from the linearity above $5.0 \times$ $10^{-7}$ torr due to the collisional damping of transient ICR signals. The effect of collisional damping is more severe when the magnetic field strength increases. These results substantiate the linear response of FT-ICR detection to the number of ions from the MPI in the ICR cell in the low pressure regime where the collisional damping of transient ICR signals is negligible.

\section{Product Branching Ratio Measurement}

To determine the product branching ratio, each ion was excited selectively by a single resonant rf pulse and the resonant peak height was measured as a function of the if pulse duration at two different trapping voltages $(0.00$ and $1.50 \mathrm{~V})$. ICR signals were acquired at $0.383 \mathrm{~T}$ with a 100 -megasamples per second transient digitizer for $320 \mu$ s. Figure 8 shows the results for $\mathrm{HCO}^{+}$and $\mathrm{CH}_{3} \mathrm{CO}^{+}$. (a)

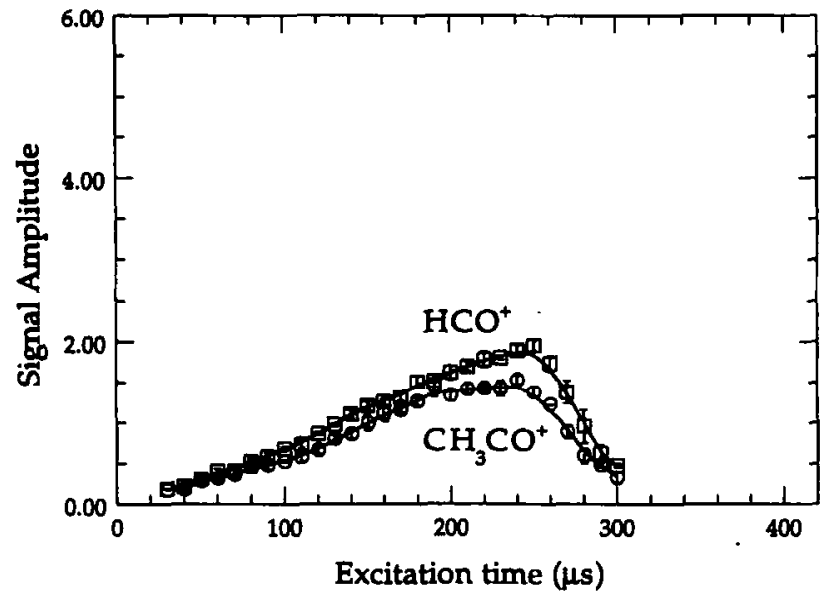

(b)

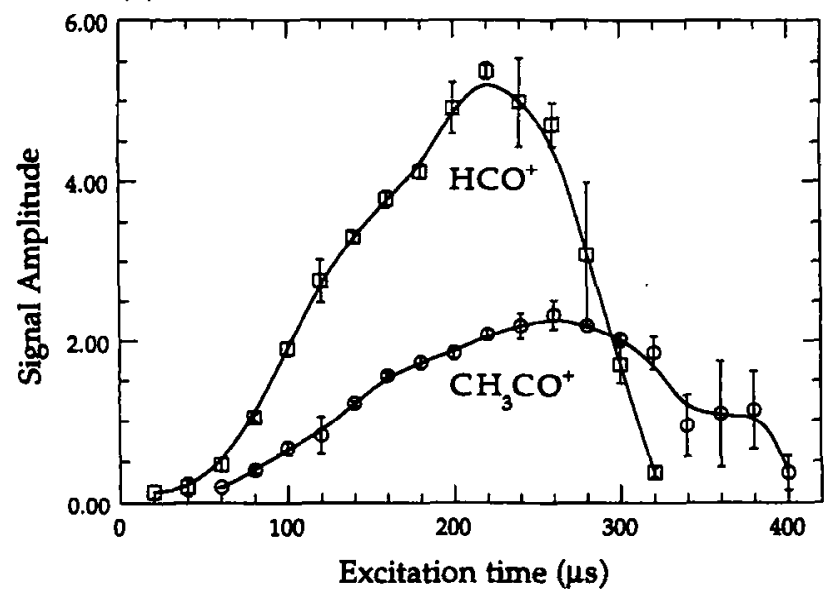

Figure 8. FT-ICR signal amplitudes as a function of the rf pulse duration. The trapping voltage was (a) $0.00 \mathrm{~V}$ and (b) $1.50 \mathrm{~V}$. The magnetic field strength was fixed at $0.383 \mathrm{~T}$. Error bars indicate standard deviations from four independent measurements.

The cyclotron radius from the resonant excitation is linearly proportional to both the amplitude and the duration of the rf pulse, but inversely proportional to the magnetic field strength as given in eq 2 :

$$
R_{\mathrm{c}}=\frac{\gamma V_{\mathrm{pP}}}{4 a B} T
$$

where $R_{c}$ denotes the cyclotron radius of the ion and $\gamma$ is the geometry parameter for the dipolar excitation, $\gamma=1.4433 ; V_{\mathrm{pp}}$ is the rf peak-to-peak amplitude, $a$ is the edge length of the ICR cell, $B$ is the magnetic field strength, and $T$ is the duration of the resonant rf pulse. The cyclotron radius is independent of mass to first order so that the peak height is free from mass discrimination. However, as noted by Xiang and Marshall [42], the resonant peak height varies with the axial and radial distributions of ions due to nonlinearities in the dipolar excitation and detection. Thus, a space-charge induced broadening of axial and radial distributions of ions needs to be suppressed as much as possible to keep ions within a linear detection regime near the 
center of the ICR cell. The space-charge density was minimized as described in the previous section by ionizing a diluted molecular beam with low laser power. Both the $\mathrm{HCO}^{+}$and $\mathrm{HC}_{3} \mathrm{CO}^{+}$ions were arrested by a zero trapping potential as shown in Figure $8 \mathrm{a}$. Their peak heights increase monotonically with increasing if pulse duration until the resonant excitation ejects ions out of the cell. Note that daughter ions arrested for $320 \mu \mathrm{s}$ in a zero trapping potential possess axial kinetic energies less than $1 \mathrm{meV}$. When a $1.50-\mathrm{V}$ trapping voltage is applied, the $\mathrm{HCO}^{+}$peak rises sharply, reaches a maximum in a shorter rf pulse duration than $\mathrm{CH}_{3} \mathrm{CO}^{+}$, and then falls off precipitously. The difference in temporal profile may be due to dissimilar spatial distributions. AKERDs shown in Figure 6 suggest that daughter ions arrested by a $1.5-\mathrm{V}$ trapping voltage are distributed within a linear detection regime located in the center half of the ICR cell along the axial direction. The radial distributions of daughter ions are determined primarily via an ionizing volume by a tightly focused laser beam for the magnetron radii and by the radial kinetic energy releases from photofragmentations for the cyclotron radii. The spatial distributions of ions are considered to be well confined to the linear detection regime in the ICR cell; thus they have little affect on the product branching ratio. The ratio of peak maxima in Figure $8 b$ yields $\left[\mathrm{HCO}^{+}\right] /\left[\mathrm{CH}_{3} \mathrm{CO}^{+}\right]=\sim 2.2 \pm 0.2$. The broadband detection in the pulsed experiment gives the branching ratio $\sim 2.1 \pm 0.2$, which is in fair agreement with that from the single resonant detection. Meanwhile, the branching ratio $\left[\mathrm{HCO}^{+}\right] /\left[\mathrm{CH}_{3} \mathrm{CO}^{+}\right]=\sim 1.6 \pm 0.1$, obtained by using a flowing sample of $5.0 \times 10^{-7}$ torr, shows a bias against the low mass ion due to the collisional damping. Under the bulk condition of flowing sample, the product branching ratio remains nearly constant over the power range 2-10 mJ/pulse. Previous quadrupole mass spectrometric studies yielded a branching ratio of 2.8 [15]. This value is somewhat higher than the present result because $\mathrm{CH}_{3} \mathrm{CO}^{+}$is depleted by sequential fragmentations to $\mathrm{CH}_{3}^{+}$and $\mathrm{CH}_{2}^{+}$. Combination of all the fragment ions derived from $\mathrm{CH}_{3} \mathrm{CO}^{+}$leads to a branching ratio of 2.0 , which is in good agreement with the present result. No reported branching ratio obtained by TOF-MS is available for comparison. Because the acetaldehyde molecular ion dissociates faster than $10^{-6} \mathrm{~s}$, the effect of kinetic shift on the appearance of the daughter ion is almost absent in the quadrupole mass spectrometric experiment.

\section{Conclusion}

The FT-ICR detection allows all of the product ions from multiphoton ionization-dissociation processes to be trapped in the absence of ion extraction fields. This complete trapping permits measurements of axial kinetic energy distributions of product ions and their branching ratios in the absence of kinetic shifts. The pressure dependence measurement shows that the product branching ratio is prone to be biased against the low mass ion because the high frequency ICR signal is subject to stronger collisional damping. The unbiased product branching ratio is measured from the collision-free transient detection by using a pulsed molecular beam. Thus FT-ICR spectrometry finds a new opportunity for studies of mass-selected MPI spectroscopy.

\section{Acknowledgments}

This work was supported by the Arnold and Mabel Beckman Foundation Young Investigator Award, the National Science Foundation (Grant CHE-9302959), and the National Science Foundation Young Investigator Award (CHE-9457668).

We acknowledge the Donors of the Petroleum Research Fund (Grant 25423-G3), administered by the American Chemical Society, for the partial support of this research. A new FT-ICR data system was purchased with support from the Camille and Henry Dreyfus Foundation Special Grant. This work also was made possible by the Santa Barbara Laser Pool under NSF Grant CHE-9413030. Special thanks go to Professor Mike Bowers for loan of the ICR setup.

\section{References}

1. Friedrich, D. M.; McClain, W. M. Ann. Rev. Phys. Chem. 1980, 31, 559-577.

2. Johnson, P. M.; Otis, C. E. Ann. Rev. Phys. Chem. 1981, 32, 139-157.

3. Lin, S. H.; Fujimara, Y.; Neusser, H. J.; Schlag, E. W. Multiphoton Spectroscopy of Molecules; Academic: New York, 1984.

4. Gobeli, D. A.; Yang, J. J.; El-Sayed, M. A. Chem. Rev. 1985, 85, 529-554.

5. Hudgens, J. W. In Advances in Multiphoton Processes and Spectroscopy; Lin, S. H. Ed.; World Scientific: Singapore, 1988, Vol. 4, pp. 171-296.

6. Ashford, M. N. R.; Howe, J. D. Ann. Reo. Phys. Chem. 1994, 45, 57-82.

7. Grotemeyer, J.; Boesl, U.; Walter, K.; Schlag, E. W. Org. Mass Spectrom. 1986, 21, 595-597.

8. Hahn, J. H.; Zenobi, R.; Zare, R. N. Science 1988, 239, 1523-1525.

9. Liang, L.; Lubman, D. M. Int. J. Mass Spectrom. Ion Processes $1989,88,197-210$.

10. Maechling, C. R.; Clemett, S. J.; Zare, R. N. Chem. Phys. Lett. 1995, 241, 301-310.

11. Schlag, E. W., Ed.; Int. J. Mass Spectrom. Ion Processes 1994, $131,1-414$.

12. Ingendoh, A.; Karas, M.; Hillenkamp, F.; Giessmann, U. Int. J. Mass Spectrom. Ion Processes 1994, 131, 345-354.

13. Reiley, J. S.; Baer, T. Int. J. Mass Spectrom. Ion Processes 1994, 131, 295-306.

14. Hwang, H. J.; Griffiths, J.; El-Sayed, M. A. Int. J. Mass Spectrom. Ion Processes 1994, 131, 265-282.

15. Fisanick, G. J.; Eichelberger, T. S.; Heath, B. A.; Robin, M. B. J. Chem. Phys. 1980, 72, 5571-5580.

16. Irion, M. P.; Bowers, W. D.; Hunter, R. L.; Rowland, F. S.; McIver, R. T., Jr. Chem. Phys. Lett. 1982, 93, 375-379.

17. Carlin, T. J.; Freiser, B. S. Anal. Chem. 1983, 55, 955-958.

18. Sack, T. M.; McCrery, D. A.; Gross, M. L. Anal. Chem. 1985, 57, 1290-1295.

19. Zimmerman, J. A.; Watson, C. H.; Eyler, J. R. Anal. Chem. 1991, 63, 361-365. 
20. Shand, N. C.; Ning, C.-L.; Pfab, J. Chem. Phys. Lett. 1995, 247, 32-37.

21. Buntine, M. A.; Metha, G. F.; McGilvery, D. C.; Morrison, R. J. S. J. Mol. Spectros. 1994, 65, 12-31.

22. Xing, X.; McDiarmid, R.; Philis, J. G.; Goodman, L. J. Chem. Phys. 1993, 99, 7565-7573.

23. Beggs, C. G.; Kuo, C.-H.; Wyttenbach, T.; Kemper, P. R.; Bowers, M. T. Int. J. Mass Spectrom. Ion Processes 1990, 100, 397-422.

24. Wyttenbach, T.; Beggs, C. G.; Bowers, M. T. Chem. Phys. Lett. 1991, 177, 239-246.

25. Kuo, C. H.; Wyttenbach, T.; Beggs, C. G.; Kemper, P. R.; Bowers, M. T. J. Chem. Phys. 1990, 92, 4849-4855.

26. Wyttenbach, T.; Bowers, M. T. J. Phys. Chem. 1992, 97, 9573-9577.

27. Heath, B. A.; Robin, M. B.; Kuebler, N. A.; Fisanick, G. J.; Eichelberger, T. S. J. Chem. Phys. 1980, 72, 5565-5570.

28. Eichelberger, T. S.; Fisanick, G. J. J. Chem. Phys. 1981, 74, 5962-5970.

29. Fisanick, G. J.; Eichelberger, T. S. J. Chem. Phys. 1981, 74, 6692-6699.

30. Gu, H.; Kundu, T.; Goodman, L. J. Phys. Chem. 1993, 97, 7194-7200.
31. Shin, S. K.; Kim, B.; Haldeman, J. G.; Hans, S.-J. J. Phys. Chem. 1996, in press.

32. Chadwick, D.; Katrib, A. J. Electron Spectrosc. 1974, 3, 39-40.

33. Cvitaš, T.; Güsten, H.; Klasinc, L. J. Chem. Phys. 1976, 64, 2549.

34. Kimura, K.; Katsumata, S.; Achiba, Y.; Yamazaki, T.; Iwata, S. S. Handbook of HeI Photoelectron Spectra of Fundamental Organic Molecules; Halsted Press: New York, 1981.

35. Creighton, J. S.; Bell, S. J. Mol. Spectrosc. 1985, 112, 285-303.

36. Neusser, H.-J.; Krause, H. Int. J. Mass Spectrom. Ion Processes 1994, 131, 211-232.

37. Dunbar, R. C.; Weddle, G. H. J. Phys. Chem. 1988, 92, 5706-5709.

38. Bloom, M.; Riggins, M. Can. J. Phys. 1974, 52, 436-455.

39. Rosenstock, H. M.; Draxl, K.; Steiner, B. W.; Herron, J. T. J. Phys. Chem. Ref. Data 1977, 6, Suppl. 1.

40. Adams, N. G.; Smith, D.; Tichy, M.; Javahery, G.; Twiddy, N. D.; Ferguson, E. E. J. Chem. Phys. 1989, 91, 4037-4042.

41. Szulejko, J. E.; McMahon, T. B. J. Am. Chem. Soc. 1993, 115, 7839-17848.

42. Xiang, X.; Marshall, A. G. J. Am. Soc. Mass Spectrom. 1994, 5, 807-813. 